\title{
Assessment of Various Physiological Fluids in Diabetes Diagnosis-A Mini Review
}

ISSN: 2637-7764

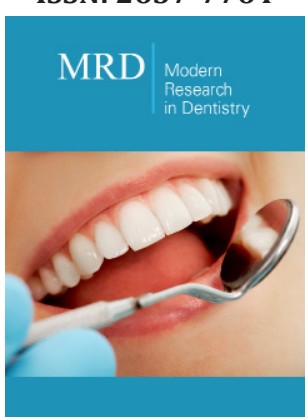

*Corresponding author: Anuradha G, Prof \& Head, Dept of Oral Medicine \& Radiology, Madha Dental College, Chennai, India

Submission: 韭June 08, 2020

Published: 海 August 12, 2020

Volume 5 - Issue 3

How to cite this article: Anuradha $G$ Arvind Muthukrishnan, Vishnupriya Veeraraghavan, Gautham Kumar N. Assessment of Various Physiological Fluids in Diabetes Diagnosis-A Mini Review. Mod Res Dent. 5(3). MRD. 000613. 2020. DOI: 10.31031/MRD.2020.05.000613

Copyright@ Anuradha G, This article is distributed under the terms of the Creative Commons Attribution 4.0 International License, which permits unrestricted use and redistribution provided that the original author and source are credited.

\author{
Anuradha $\mathrm{G}^{1 *}$, Arvind Muthukrishnan², Vishnupriya Veeraraghavan ${ }^{3}$ and \\ Gautham Kumar $\mathbf{N}^{4}$ \\ ${ }^{1}$ Prof \& Head, Dept of Oral Medicine \& Radiology, Madha Dental College, Chennai, India \\ ${ }^{2}$ Prof \& Head, Dept of Oral Medicine \& Radiology, Saveetha Dental College, Chennai, India \\ ${ }^{3}$ Prof, Dept of Biochemistry, Sabetha Dental College, Chennai, India \\ ${ }^{4}$ Prof, Dept of Periodontics \& Implantology, Madha Dental College \& Hospital, Chennai, India
}

\section{Abstract}

Diabetes mellitus is a metabolic disorder characterized by hyperglycemia due to insulin deficiency or its ineffectiveness. Successful management of diabetes involves constant monitoring of the glycemic status of the patient which is usually done by estimating the glucose concentration in blood. However, drawing blood is always an invasive procedure which has prompted researchers to look at alternative biofluids as a reliable substitute to blood for glucose estimation. This mini review examines various biofluids including urine, tears, sweat, and saliva as possible candidates for glucose estimation in diabetic patients.

\section{Introduction}

Diabetes mellitus is a group of metabolic diseases characterized by hyperglycemia resulting from an absolute deficiency of insulin secretion and/or reduction in the biological effectiveness of insulin or both [1]. Due to the burden of this disease across the globe, diabetes mellitus is considered as one of the priority non communicable diseases and various measures have been put forward for an effective screening, diagnosing and monitoring the diabetic status in patients [2]. The classic symptoms of untreated diabetes are unintended weight loss, polyuria, polydipsia, polyphagia and other symptoms of diabetes include tiredness, blurred vision, fatigue, headache, slow healing of wounds and itchy skin. Diabetic retinopathy, neuropathy, nephropathy and microvascular angiopathy leading to cardiovascular diseases are long term complications [3]. The estimation of blood sugar levels have always been the gold standard in estimating the diabetic status of individuals. Since blood testing is invasive, painful and also leads to anxiety, risk of infection and also needs a skilled phlebotomist to withdraw blood, blood testing may result in noncompliance with healthcare services to perform a blood test to diagnose or monitor the glycaemia status in patients [4]. So recent advances focus on various biological fluids other than blood in diagnosing and monitoring the blood glucose level in patients [5]. Since 1841, urine has been used as a diagnostic fluid for diabetes assessment. It has been extensively studied as it is very easy to collect and is composed of metabolites such as glucose, proteins and other dissolved salts [6]. Glucose can be found in urine when it is excreted from blood in elevated levels and as a result, this fluid has been investigated for the diagnosis of diabetes [7]. But the main disadvantage of using urinary glucose in diagnosis and screening of diabetes mellitus includes marked individual variations in the renal threshold for glucose, poor reflection of changing levels of hyperglycemia and lack of specificity and sensitivity of various qualitative and semi quantitative procedures [8]. The other physiological fluid used in monitoring glucose levels is the interstitial fluid, Blood and surrounding vascularized tissue readily exchange biological analytes and small molecules by diffusion with the interstitial fluid. Methods of monitoring glucose via the skin has become popular and counteract the challenges associated with patient compliance and invasive monitoring. The Glucowatch was developed as a wearable device which used reverse iontophoresis to extract interstitial fluid through skin and measure glucose levels [9]. 
Although Glucowatch was a considerable advancement towards noninvasive and continuous glucose monitoring, the approach was hampered by the need of periodic recalibration, thereby resulting in increase in cost for testing equipment and patient care. Other drawbacks include long warm up times, sweating and skin rash with irritation which subsequently resulted in product removal from the market [10]. Sweat was also tried for diagnostic purposes in diabetes mellitus but the analytes contained in sweat varied significantly between basal and exercising states as well as between individuals [11]. There are various researches including eye glasses bio sensor system, watch sensing platform for glucose sensing in sweat, glucose sensor integrated into a wearable wrist band for monitoring glucose levels using sweat. Although sweat sensing for diagnosis is very promising there are also some concerns associated with this sensing fluid [12]. The main challenges include limited fundamental knowledge about this sensing fluid compared to blood, sampling issues associated with sweat production by exercising, surface contamination due to skin impurities and variability in the rate of sweat production. Ocular fluids have also been analysed and this fluid is excreted from the body in the form of tears. Analytes found in this fluid includes glucose, ascorbic lactate, proteins, hormones and can offer great insight into individuals health status [13].

As a result, ocular fluid was investigated for noninvasive and continuous glucose monitoring. A smart contact lens was created and was correlated with the blood glucose levels in diabetic patients. Using contact lens as a sensing platform had many advantages including real time continuous and noninvasive glucose monitoring. However, the disadvantages include production of corrosive hydrogen peroxide as a bi product in the electrochemical sensing approach used in the smart lens and blinking causing artefact in the sensor signal. The human saliva, an exocrine fluid secretion has high potential for screening health and diseases. It consists of water, electrolytes and a variety of proteins, polypeptides, and also studies have shown that the proteins present in blood are present in saliva as well [14]. Therefore, saliva is functionally comparable to blood in reflecting the physiological status of the body [15]. The glucose levels present in saliva correlates directly with blood glucose levels. However, the mechanism of secretion is not very clear. Many authors have explained various possible mechanisms to explain increased glucose content in saliva of diabetic patients. There is no single mechanism to explain the appearance of glucose in saliva during periods of prolonged hyperglycemia. The small molecular size, possible damage in the permeability of basement membrane, changes in the blood vessels, increased leakage from the ductal cells and leakage through the gingival crevices all may contribute to the multifactorial cause of increased levels of saliva glucose in diabetics. Saliva as a diagnostic fluid has many advantages of being a simple, noninvasive method and diagnosis of disease with analysis of saliva is potentially valuable since collection of this fluid is associated with fewer compliance problems as compared to blood. No special equipment is needed for collection of fluid Saliva is a promising biofluid for early disease detection that leads to more effective treatment, risk assessment for estimation of glucose level.

\section{Conclusion}

Blood has always been a standard for screening, monitoring, and diagnosing diabetes. However, saliva transcends several of its disadvantages and fulfils the diagnostic concerns as it closely reflects blood levels. Saliva testing surpasses all the limitations faced by various biological fluids in evaluating the glucose levels in diabetic patients.

\section{References}

1. Malamed Stanley F (2015) Medical emergencies in the dental office. $\left(7^{\text {th }}\right.$ Edn), Mosby, Missouri, USA.

2. WHO (2019) Global report on diabetes. World Health Organization, Geneva, Switzerland.

3. Rockefeller JD (2015) Diabetes: symptoms, causes, treatment and prevention.

4. Tura A, Maran A, Pacini G (2007) Non-invasive glucose monitoring: Assessment of technologies and devices according to quantitative criteria. Diabetes Res Clin Pract 77(1): 16-40.

5. Sokolowski CJ, Giovannitti JA, Boynes SG (2010) Needle phobia: etiology, adverse consequences and patient management. Dent Clin North Am 54(4): 731-744.

6. Zhang W, Du Y, Wang ML (2015) On chip highly sensitive saliva glucose sensing using multilayer films composed of single-walled carbon nanotubes, gold nano particles and glucose oxidase. Sensing and BioSensing Research 4: 96-102.

7. Yao H, Shum A J, Cowan M, Lahdesmaki I, Parvis BA (2011) A contact lens with embedded sensor for maintaining tear glucose level. Biosensors and Bioelectronics 26(7): 3290-3296.

8. Reinauer H, Home PD, Kanagasabapathy AS, Heuck CC (2002) Laboratory diagnosis and monitoring of diabetes mellitus. WHO, Geneva, Switzerland.

9. Fang G, Kaur G, Wang B (2004) Progress in boronic acid-based fluorescent glucose sensors. Journal of Fluorescence 14(5): 481-489.

10. Yoo EH, Lee SY (2010) Glucose biosensors: An overview of use in clinical practice. Sensors 10(5): 4558-4576.

11. Heikenfeld J (2016) Noninvasive analyte access and sensing through eccrine sweat: challenges and outlook circa 2016. Electroanalysis 28(6): $1242-1249$

12. Bandodkar AJ, Wang J (2014) Non-invasive wearable electrochemical sensors: a review. Trends in Biotechnology 32(7): 363-371.

13. Viswanath B, Choi CS, Lee K, Kim S (2017) Recent trends in the development of diagnostic tools for diabetes mellitus using patient saliva. TrAC Trends in Analytical Chemistry 89: 60-67.

14. Wang Q Yu Q, Lin Q, Duan Y (2015) Emerging salivary biomarkers by mass spectrometry. Clinica Chimica Acta 438: 214-221.

15. Abikshyeet P, Ramesh V, Oza N (2012) Glucose estimation in the salivary secretion of diabetes mellitus patients. Diabetes Metab Syndr Obes 5: $149-154$. 